\title{
Comparison of Repetitive Control Schemes for a DC Servo Motor
} Jacob S Glower*

Department of Electrical Engineering, North Dakota State University, Fargo, North Dakota, USA

\begin{abstract}
In this paper, four types of repetitive controllers are compared on a DC servo motor. It is shown that a Fouriertype repetitive controller is superior in terms of the adaptation rate and, for tracking a sine wave and a half-rectified sine wave.
\end{abstract}

Keywords: DC servo motor; PIC microcontroller; Repetitive control schemes

\section{Introduction}

Since repetitive controllers were first proposed [1], several variations have been proposed [2]. The four of which the author is aware include the following:

\section{Repetitive Control}

Each cycle is broken down into $\mathrm{N}$ time-slots. The ideal input (defined as the input required to force the desired motion) is then approximated as a constant during each time slot. The adaptive controller estimates these $\mathrm{N}$ constants.

\section{Bilinear Repetitive Control}

Instead of approximating the ideal input as a constant during each time slot, it is approximated as a line:

$$
U_{\text {ideal }}=c_{0}+c_{1} \tau
$$

where $\tau$ measures the time into the present time slot going from 0 to 1 . By using a more accurate approximation, better tracking should result at the cost of estimating more parameters.

\section{Polynomial Repetitive Control}

Still further improvements in tracking accuracy can be obtained by using higher-order polynomials during each time slot:

$$
U_{\text {ideal }}=c_{0}+c_{1}+c_{2} \tau^{2}
$$

\section{Fourier Repetitive Control}

Alternatively, since the ideal input is periodic, it can be expressed in terms of its Fourier series:

$$
U_{\text {ideal }}=b_{0}+\sum\left(\left(a_{n} \sin (n \omega t)+b_{n} \cos (n \omega t)\right)\right.
$$

where $\omega$ is the fundamental frequency of the periodic motion.

With four different schemes being proposed, one may wonder which scheme is superior. In terms of their long-term performance using an infinite number of terms, each is identical. As proven, asymptotic perfect model following can be assured using adaptive control techniques so long as the ideal input can be expressed as a known function matrix or basis function, $\mathrm{W}(\mathrm{t})$, times a constant but unknown matrix [3-11], K:

\section{Uideal $=\mathrm{W}(\mathrm{t}) \mathrm{K}$}

Each of the proposed repetitive control schemes falls into this category. For example, $\mathrm{W}(\mathrm{t})$ for a repetitive controller consists of $\mathrm{N}$ delta functions: one per time slot. Similarly, $\mathrm{W}(\mathrm{t})$ for a Fourier repetitive controller consists of the harmonics of the repetition frequency.

Since each of the proposed repetitive control schemes can be described, each assures asymptotic perfect tracking. Likewise, any comparison must be based upon the performance in finite time and/ or using a finite dimensional $\mathrm{W}(\mathrm{t})$ and $\mathrm{K}$. Such a comparison is made in this paper.

This paper is organized as follows:

- In Section II, the DC servo motor is presented along with the repetitive control law with a generic basis function is derived for a DC servo motor.

- In Section III, the effect of using a finite number of terms on the tracking accuracy is presented. Unfortunately, this accuracy depends upon the particular motor and the particular path being followed. For the sake of comparison, therefore, two waveforms are selected: a $1 \mathrm{~Hz}$ sine wave and a $1 \mathrm{~Hz}$ half-wave rectified sine wave.

- In Section IV, results and conclusions are presented.

\section{Hardware and Repetitive Control}

Hardware set up

Clifton DC Servo Motor AMC 30A8T power amplifier PIC microcontroller.

\section{Set Point}

$0.5 \mathrm{~Hz}$ Sine Wave (best case)

$0.5 \mathrm{~Hz}$ Half Rectified Sine Wave (impulse in 2nd derivative).

\section{Repetitive control}

The ideal input is estimated using hyperstability-based MRAC [12]. For a DC servo motor, the dynamics are approximately:

$$
\theta \approx\left(\frac{100}{s(s+8)}\right) V_{a}
$$

*Corresponding author: Jacob S Glower, Department of Electrical Engineering North Dakota State University, Fargo, North Dakota, USA, Tel: 7012318068 E-mail: jacob.glower@ndsu.edu

Received July 14, 2017; Accepted August 04, 2017; Published August 14, 2017 Citation: Glower JS (2017) Comparison of Repetitive Control Schemes for a DC Servo Motor. Adv Robot Autom 6: 168. doi: 10.4172/2168-9695.1000170

Copyright: (c) 2017 Glower JS. This is an open-access article distributed under the terms of the Creative Commons Attribution License, which permits unrestricted use, distribution, and reproduction in any medium, provided the original author and source are credited. 
Citation: Glower JS (2017) Comparison of Repetitive Control Schemes for a DC Servo Motor. Adv Robot Autom 6: 168. doi: 10.4172/21689695.1000170

Page 2 of 6

If this plant is used with a PI-type parameter estimation, a type2 system will be created in the parameter estimates, resulting in a highly oscillatory system [13]. To prevent this, unity feedback is placed around the motor:

$V_{a}=(\operatorname{Ref}-\theta)$

where Ref is the set point (reference angle), resulting in

$\theta \approx\left(\frac{100}{s^{2}+8 s+100}\right) \operatorname{Ref}$

Next, to track a given waveform, the input (Ref) is defined as

$R=k 1 b 1(t)+k 2 b 2(t)+$

where $k_{i}$ are unknown constants and $b_{i}(t)$ are periodic functions that serve as the basis for the input required to force the angle to track some desired path, $\theta R$.

Hyperstability-based MRAC is used to estimate these constants as: $\left(\frac{100}{s^{2}+8 s+100}\right)\left(\frac{s+1}{s}\right)$

where the PI compensator gains were selected to keep the root locus of $\left(\frac{100}{s^{2}+8 s+100}\right)\left(\frac{s+1}{s}\right)$ negative real, assuring stability of the MRAC system [13].

This algorithm is implemented on a PIC18F4620 microcontroller with a sampling rate of $50 \mathrm{~Hz}$.

The set point to be tracked is a $1 \mathrm{~Hz}$ sine wave and a $1 \mathrm{~Hz}$ halfrectified sine wave to give a flavor for how each scheme behaves in the best case and in a fairly typical case.

The tracking error of the feedback control system with $\operatorname{Ref}=\theta R$ is show in Figures 1 and 2. Note that for each case, a significant delay exists, as is expected for an error driven device. In addition, for the halfwave rectified sine wave, the high-frequency terms have poor tracking.

\section{Tracking with MRAC and a Finite Number of Terms}

In theory, given any basis, $b i(t)$, which spans the ideal input (defined as the input required to track the set point exactly) will result in perfect tracking. With a finite number of terms, however, the tracking will be

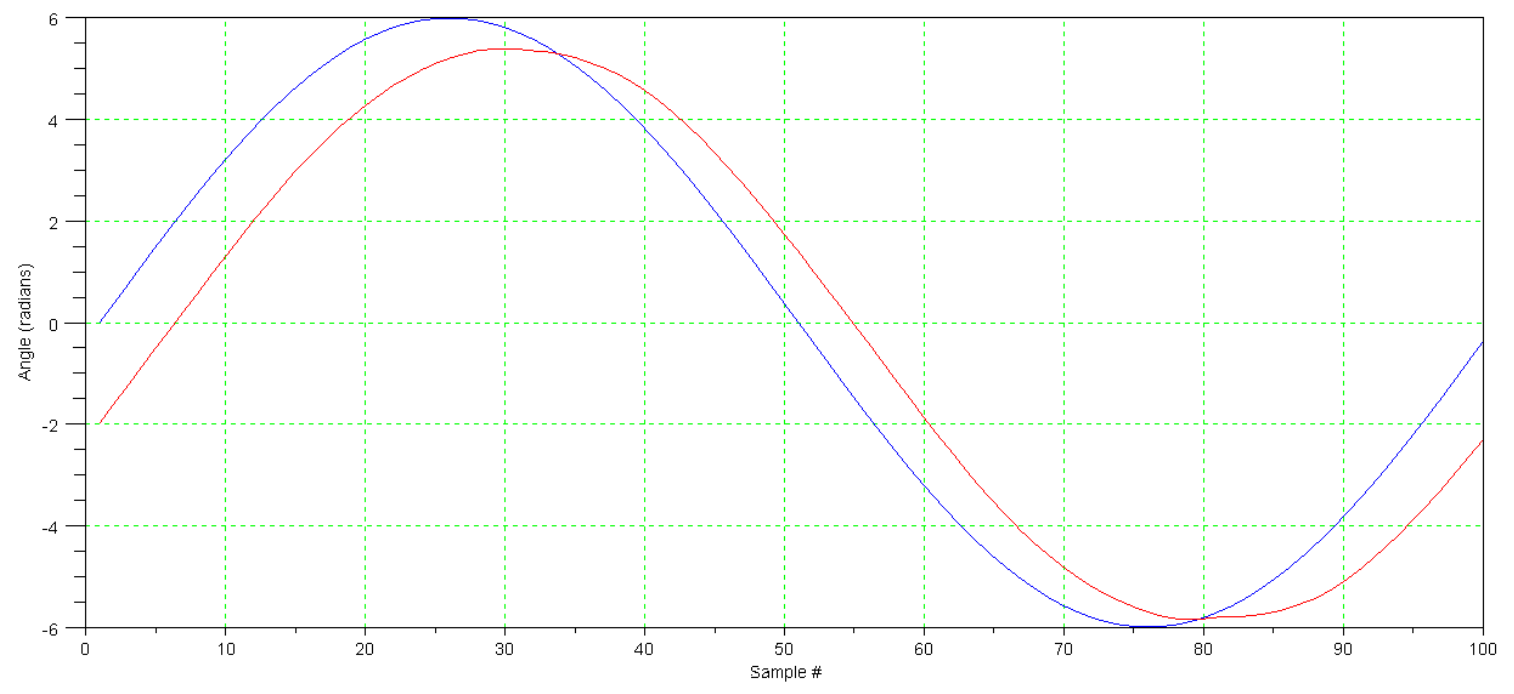

Figure 1: rms error $=0.12466$ radians

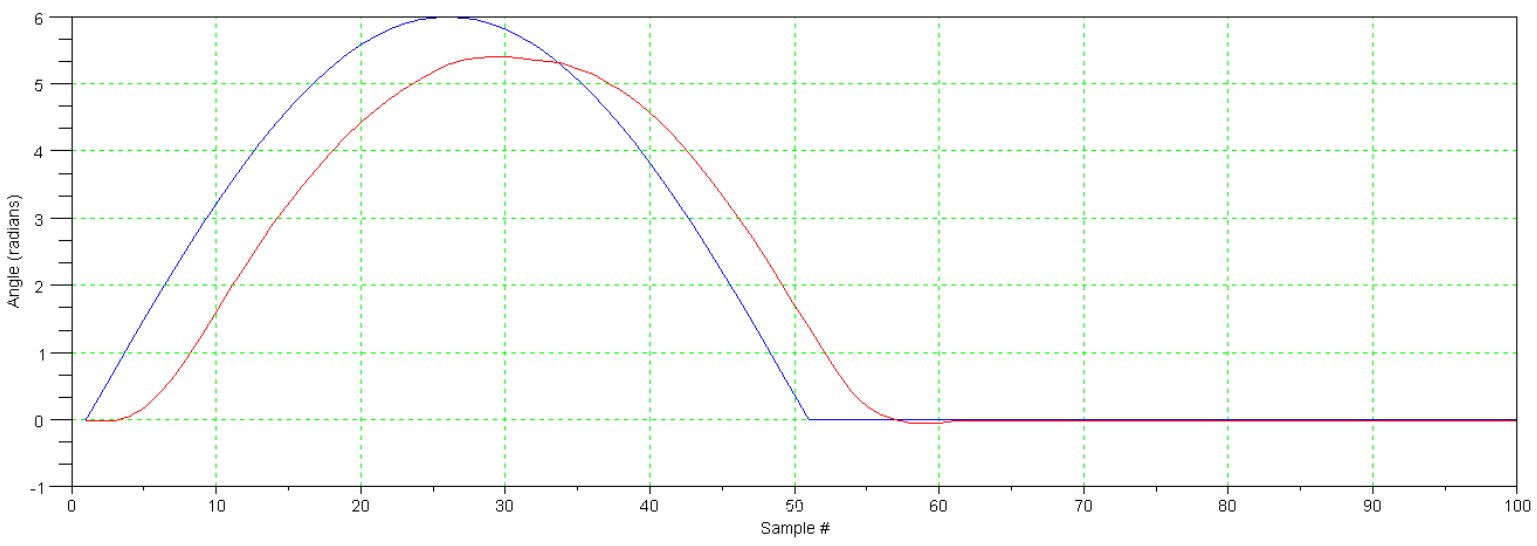

Figure 2: rms error $=0.0776009$ 
Citation: Glower JS (2017) Comparison of Repetitive Control Schemes for a DC Servo Motor. Adv Robot Autom 6: 168. doi: 10.4172/21689695.1000170

Page 3 of 6

imperfect. In this section, the result of limiting the basis to 10 or fewer terms is investigated.

MRAC: Model-reference adaptive control is used to control the motor. The idea here is as follows:

The 'ideal' input is the input required to force the output to track the set point perfectly. Since the 'ideal' input is not known a-priori, express it in terms of a basis function:

\section{Uideal $=\sum k i B i(t)$}

where $k i$ is a set of unknown constants and $\mathrm{B} i(t)$ is a set of basis functions.

Hyperstability-based model reference adaptive schemes tend to use a type of PID control with the estimation of the constants, $k i$. When trying to control a type- 1 system, this in essence creates a type- 2 system for the parameter estimates, creating stability issues. [10]. To eliminate this second integrator, a feedback loop around motor angle is created:

$$
U=k(R e f-\theta)
$$

where $\mathrm{R}$ is the set point or reference angle. Now, the 'ideal' input is the set point, $R$, which causes the motor to track the set point, $\theta R$.

For the following MRAC schemes, this technique was used as follows. The error between the set point and the actual angle is defined as:

$$
E=\theta R-\theta
$$

The reference angle is found using a set of basic functions:

$$
\operatorname{Ref}=\sum k i B i
$$

where $k i$ are unknown constants estimated using the following PI scheme:

$$
k_{i}=\left(\frac{s+1}{s}\right) E \cdot B_{i}
$$

In theory, any basis function which spans all periodic inputs can work. In practice, some are probably better than others. The tracking ability for several basis functions follow [14-16]:

\section{Repetitive control}

$B_{i}=\left\{\begin{array}{cc}1 & \text { in time slot } \mathrm{i} \\ 0 & \text { otherwise }\end{array}\right.$.

\section{Bilinear repetitive control}

MRAC sets the midpoint in each time slot. Use linear interpolation between time slots (Figures 3-6).

Problem: This isn't guaranteed stable. The basis function is a constant for each time slot (the midpoint). The actual basis interpolates between adjacent time slots.

\section{Legendre polynomials}

A Legendre polynomial is an orthonormal power series $\left(1, t, t^{2}, t^{3}\right.$,

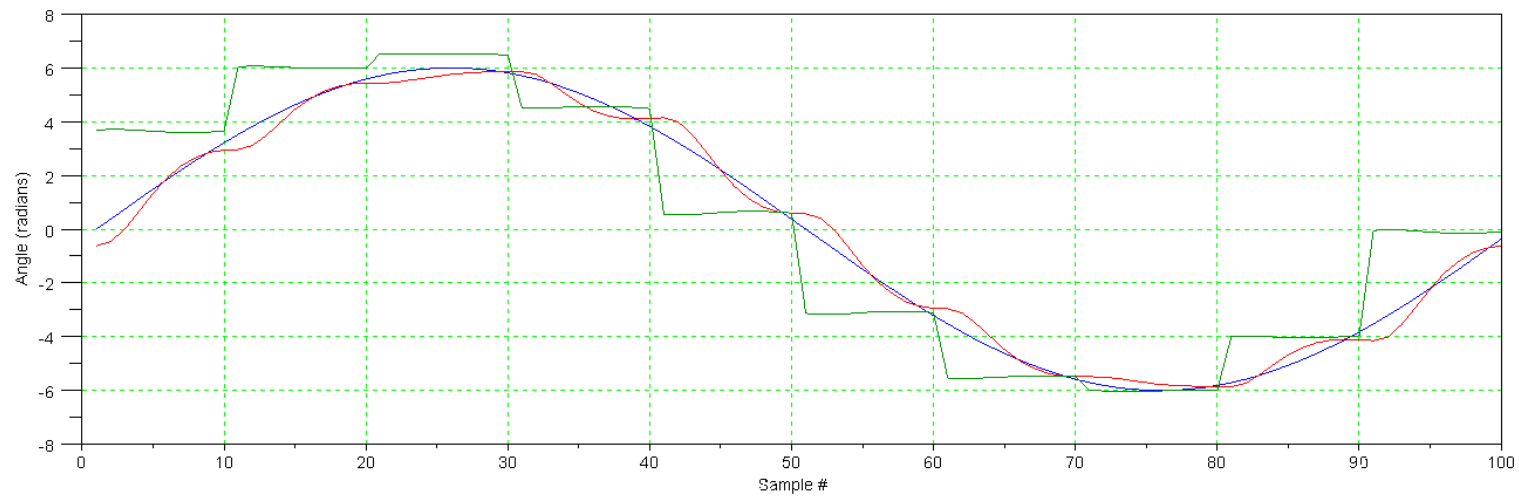

Figure 3: Ability to track a sinusoidal set point: Blue $=$ reference angle, red $=$ actual angle, green $=$ input from MRAC algorithm. $\mathrm{RMS}$ error $=0.0360065$ radians

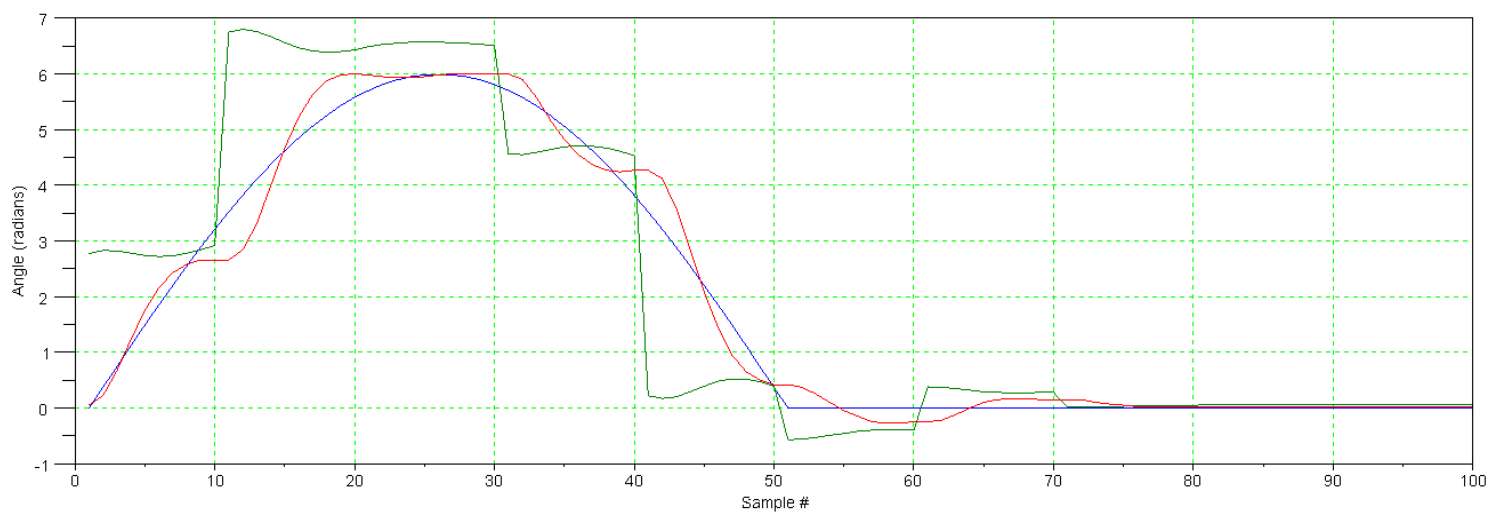

Figure 4: Ability to track a half-rectified sine wave. The RMS error is 0.0299643 radians. 
Citation: Glower JS (2017) Comparison of Repetitive Control Schemes for a DC Servo Motor. Adv Robot Autom 6: 168. doi: 10.4172/21689695.1000170

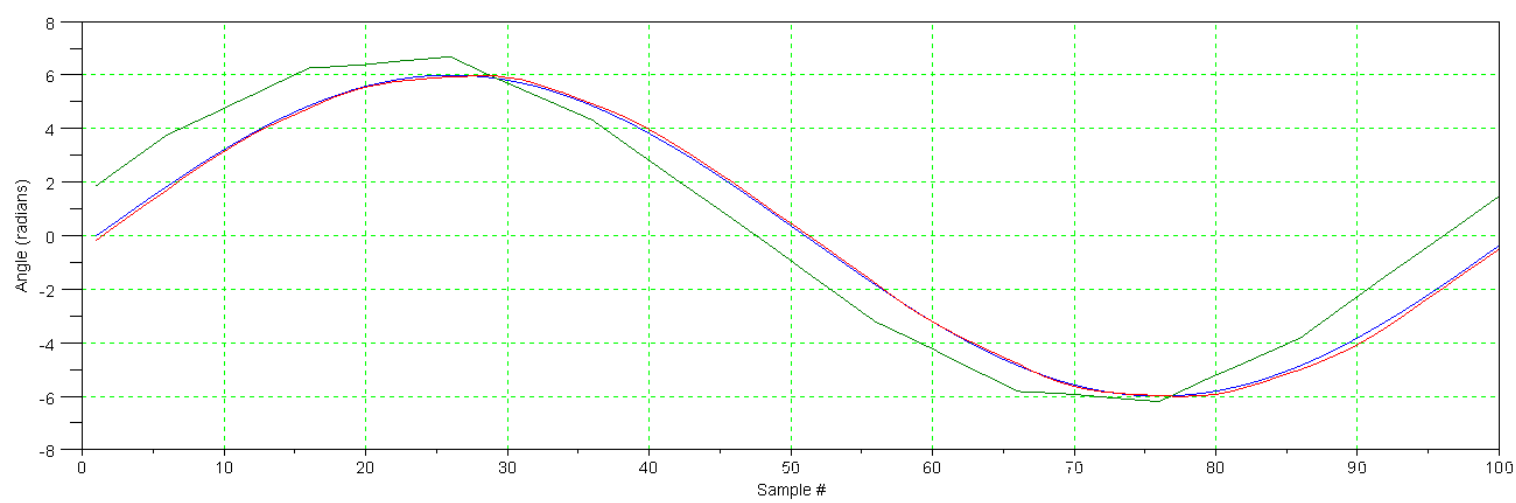

Figure 5: Tracking a sine wave using bilinear repetitive control RMS error $=0.0106850$.

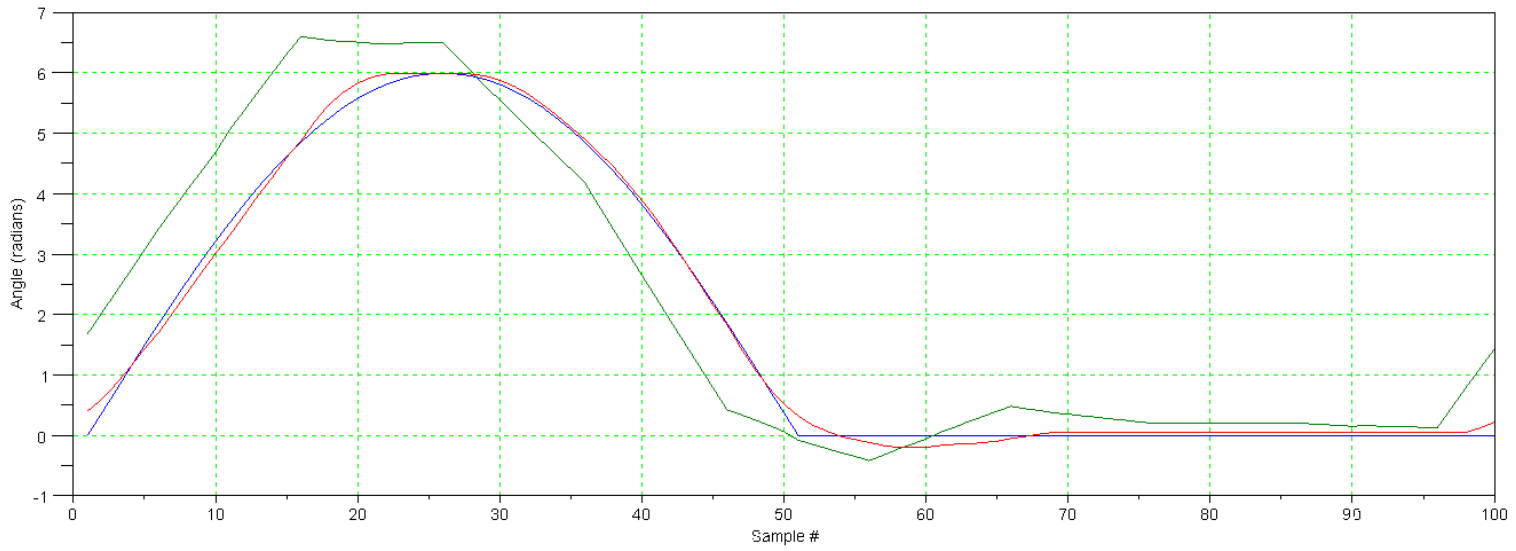

Figure 6: Tracking a half-rectified sine wave using bilinear repetitive control RMS error $=0.0119151$.

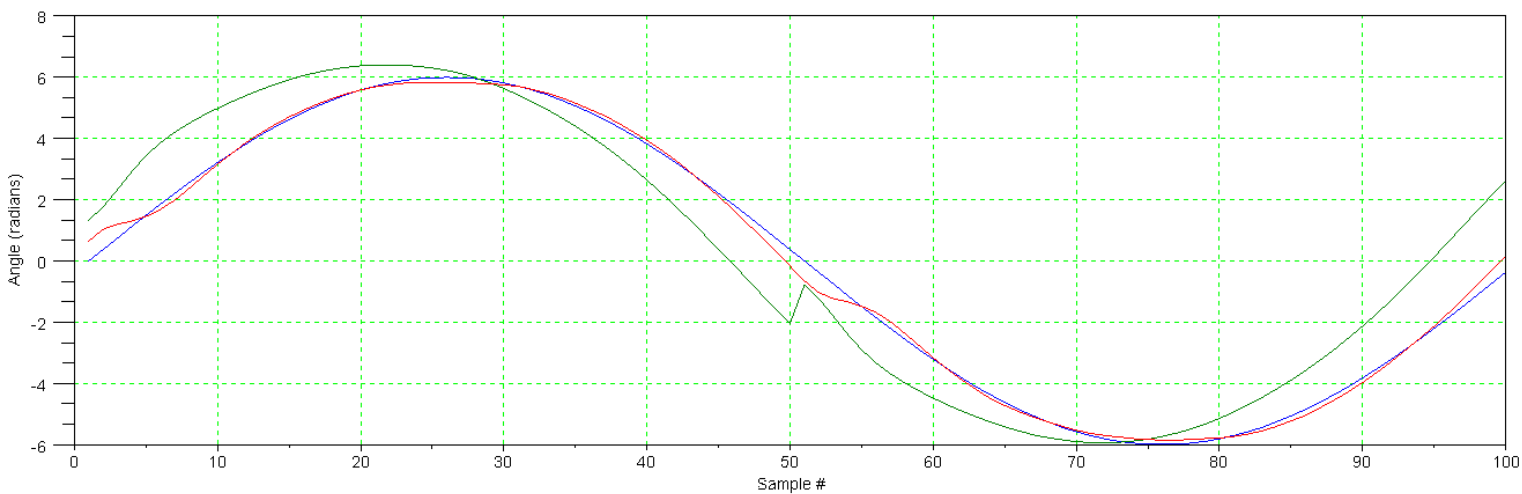

Figure 7: Tracking a sine wave using Legendre polynomials. Blue $=$ set point, red $=$ actual angle, green $=$ input $($ Ref $)$. Two regions are used: $0<t<T / 2$ and $\mathrm{T} / 2<\mathrm{t}<\mathrm{T}$. This creates the bump at $\mathrm{T} / 2$. RMS error $=0.0215202$.

...). Defining the time slot to be $t \in[-1,1]$ one valid set of basis functions in time are (Figures 7 and 8 ):

$B 0=1$

$B_{1}=1.22 t$

$$
B_{2}=1.44 t^{2}-1 \text {. }
$$

\section{Fourier repetitive}

Yet another valid basis comes from a Fourier series expansion. Since the 'ideal' input is periodic, it can be expressed in terms of it's 
Citation: Glower JS (2017) Comparison of Repetitive Control Schemes for a DC Servo Motor. Adv Robot Autom 6: 168. doi: 10.4172/21689695.1000170

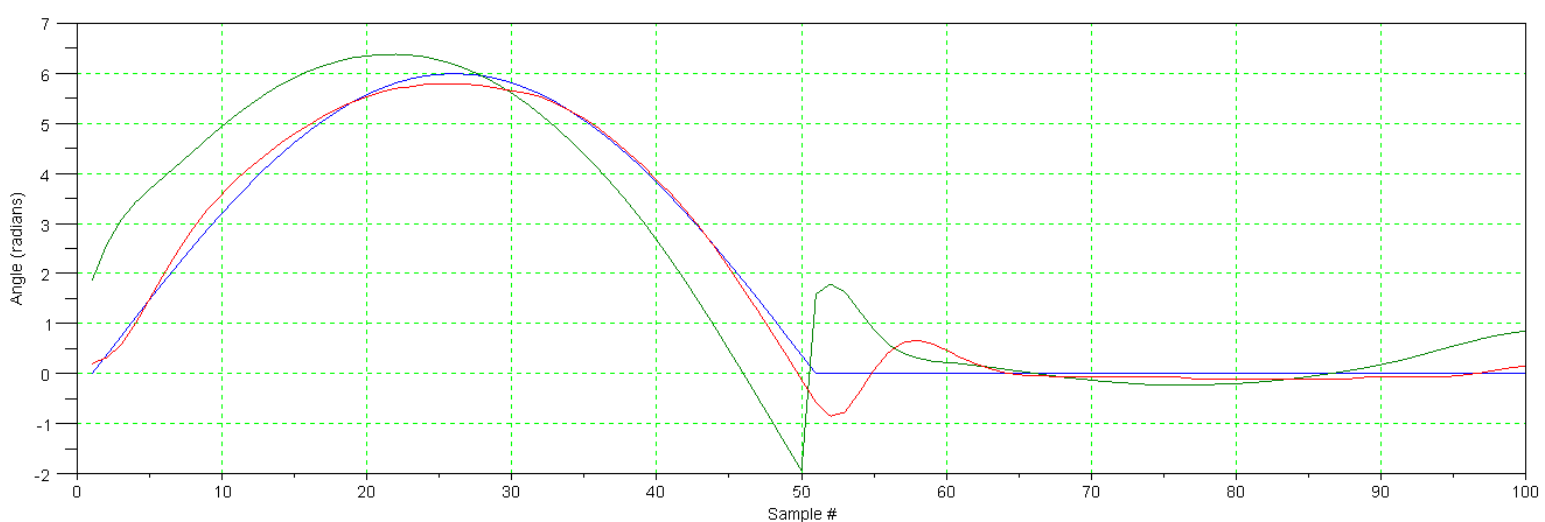

Figure 8: Tracking a half-wave rectified sine wave using Legendre polynomials. Two regions are used: $0<\mathrm{t}<\mathrm{T} / 2$ and $\mathrm{T} / 2<\mathrm{t}<\mathrm{T}$. This creates the bump at $\mathrm{T} / 2$. RMS error $=0.0239508$.

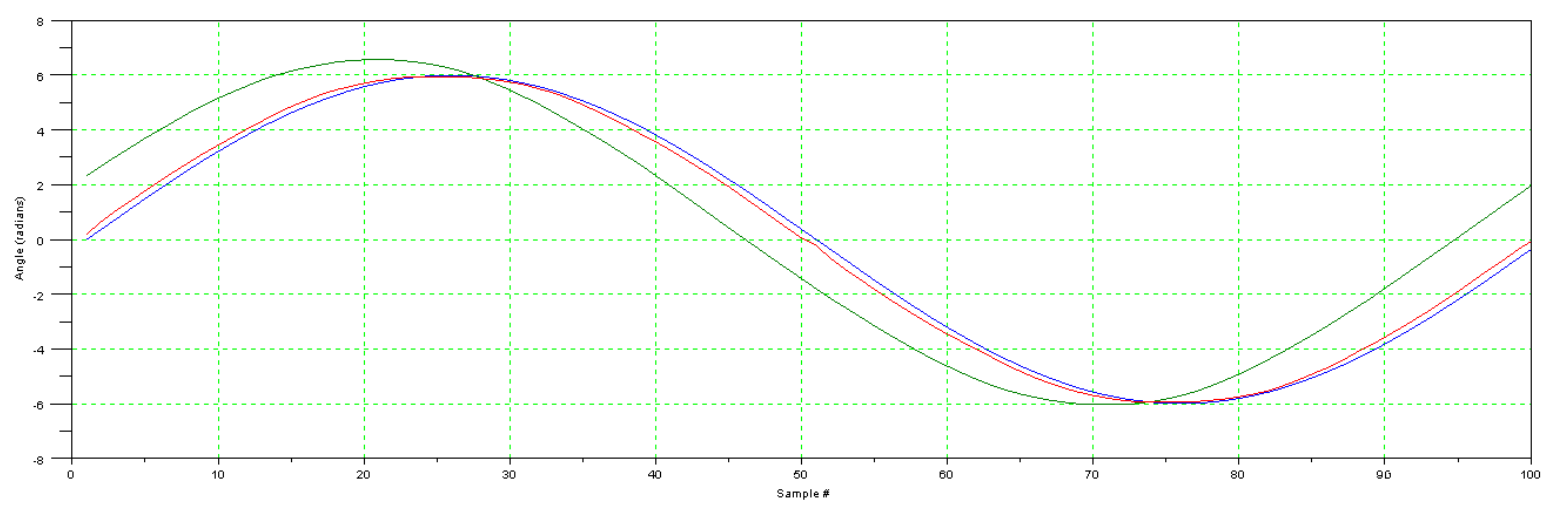

Figure 9: Tracking a sine wave using Fourier series. Blue $=$ set point, red $=$ actual angle, green $=$ input $($ Ref $)$. RMS error $=0.0219632$

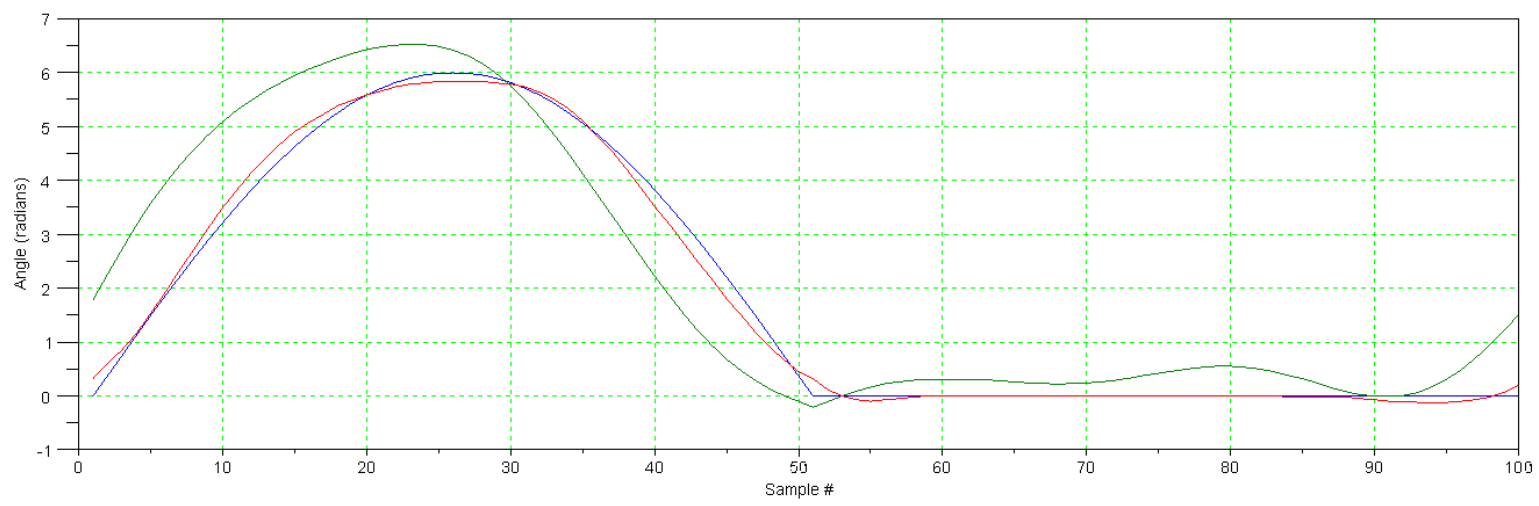

Figure 10: Tracking a half-wave rectified sine wave using a Fourier series. RMS error $=0.0158288$.

Fourier series. The truncated series used here is (Figures 9 and 10):

$B_{i}=\{1, \sin (t), \cos (t), \sin (2 t), \cos (2 t), \sin (4 t), \cos (4 t)\}$

\section{Results and Conclusions}

RMS Error (radians * 10e-3).

\begin{tabular}{|c|c|c|}
\hline Method & Sine Wave & $\begin{array}{c}\mathbf{1} / \mathbf{2} \text { Wave Rectified Sine } \\
\text { Wave }\end{array}$ \\
\hline Error Feedback & 124.66 & 77.6009 \\
\hline 10 Time Slots: Constant & 36.0065 & 29.9643 \\
\hline 10 time slots - interpolated & 10.6850 & 11.9151 \\
\hline Polynomial & 21.5202 & 23.9508 \\
\hline Fourier & 21.9632 & 15.8288 \\
\hline
\end{tabular}


Citation: Glower JS (2017) Comparison of Repetitive Control Schemes for a DC Servo Motor. Adv Robot Autom 6: 168. doi: 10.4172/21689695.1000170

\section{References}

1. Hara S, Yammato Y, Omata T, Nakano M (1988) Repetitive Control System: A new type servo system for periodic exogenous signals. IEEE Transactions on Automatic Control 3: 659-667.

2. Sadegh N, Guglielmo K (1991) A new repetitive controller for mechanical manipulators. Journal of Robotic Systems.

3. Sadegh N, Horowitz R (1987) Stability analysis of and adaptive controller for robotic manipulators. Proceedings of the 1987 IEEE Conference on Robotics and Automation, Raleigh, NC, pp: 1223-1228.

4. Sadegh N, Horowitz R, Kao W, Tomizuka M (1990) Unified approach to the design of adaptive and repetitive controllers for robotic manipulators. Transactions of the ASME 112: 618-629.

5. Sadegh N, Guglielmo K (1992) Design and Implementation of Adaptive and Repetitive Controllers for Mechanical Manipulators. IEEE Transactions on Robotics and Automation 8: 395-400.

6. Tsakalis KS, Ioannou PA (1987) Adaptive Control of Linear Time-Varying Plants: A New Controller Structure. Proceedings of the 1987 American Controls Conference, Minneapolis, MN, pp: 583-588.

7. Tsakalis KS, loannou PA (1987) Adaptive control of linear time-varying plants. Automatica 23: 459-468.

8. Tsakalis KS, loannou PA (1988) Adaptive control of time-varying plants: simple examples. The International Journal of Control and Signal Processing 2: 291 309

9. Tsakalis KS, loannou PA (1988) A new indirect adaptive control scheme for time-varying plants. Proceedings of the 27th Conference on Decision and Control, Austin, Texas, pp: 2419-2424.

10. Glower JS (1988) On the Specification of Adaptation Rates in MRAC Systems. The Ohio State University.

11. Moore JB, Horowitz R, Messner W (1992) Functional Persistence of Excitation and Observability for Learning Control Systems. Journal of Dynamic Systems, Measurement, and Control 114: 500-507.

12. Landau ID (1979) Adaptive Control: The Model Reference Approach. New York: Marcel Dekker, pp: 169-170.

13. Glower JS (1989) Adaptation rates for MRAC systems. The Internationa Journal of Control 50: 1645-1666.

14. Horowitz R, Messner W, Moore JB (1991) Exponential Convergence of a Learning Controller for Robot Manipulators. IEEE Transactions on Automatic Control 36: 890-894.

15. Narendra KS, Taylor JH (1973) Frequency Domain Criteria for Absolute Stability. New York: Academic Press.

16. Popov VM (1963) Solution of a New Stability Problem for Controlled Systems. Automation and Remote Control 24: 1-23. 